\title{
Gas exchange in fruits related to skin condition and fruit ripening studied with diode laser spectroscopy
}

\author{
Jing Huang \\ Hao Zhang \\ Huiying Lin \\ Tianqi Li \\ Liang Mei \\ Katarina Svanberg \\ Sune Svanberg
}




\title{
Gas exchange in fruits related to skin condition and fruit ripening studied with diode laser spectroscopy
}

\author{
Jing Huang, ${ }^{a}$ Hao Zhang, ${ }^{a}$ Huiying Lin, ${ }^{a}$ Tianqi Li, ${ }^{a}$ Liang Mei, ${ }^{b}$ Katarina Svanberg, ${ }^{a, b}$ and Sune Svanberg ${ }^{a, b, \star}$ \\ ${ }^{a}$ South China Normal University, Center for Optical and Electromagnetic Research, University City Campus, Research Building 5 , \\ Guangzhou 510006, China \\ bLund University, Lund Laser Center, P.O. Box 118, SE-221 00 Lund, Sweden
}

\begin{abstract}
The concentration of the biologically active molecular oxygen gas is of crucial importance for fruits in the metabolic respiration, maturation, and ripening processes. In our study, oxygen content and oxygen transport in fruits, exemplified by apples and guavas, were studied noninvasively by gas in scattering media absorption spectroscopy. The technique is based on the fact that free gases typically have 10,000 times narrower absorption features than the bulk material. The technique was demonstrated in studies of the influence of the fruit skin in regulating the internal oxygen balance, by observing the signal response of the internal oxygen gas to a transient change in the ambient gas concentration on peeled and unpeeled fruits. In addition, the gas exchange rate at different ripening stages was also studied in intact guavas. ๑2016 Society of Photo-Optical Instrumentation Engineers (SPIE) [DOI: 10.1117/1.JBO.21.12.127007]
\end{abstract}

Keywords: diode laser spectroscopy; gas in scattering media absorption spectroscopy; gas exchange; fruit skin; oxygen; respiration; ripening.

Paper 160642R received Sep. 16, 2016; accepted for publication Nov. 28, 2016; published online Dec. 23, 2016.

\section{Introduction}

Generally, for all living organisms such as fruits, the respiratory metabolism - in which the organic compounds are broken down to provide energy for life-can mainly be separated into two kinds of processes-aerobic respiration and anaerobic respiration. In the former case, oxygen is needed and carbon dioxide is produced in the process. Respiration does not consume oxygen, e.g., when inadequate oxygen exists in the surroundings the state is called anaerobic respiration. In this process, the organic compounds are still broken down but not fully into the simplest substances, while new compounds are produced, resulting in the decay of the fruits and a loss of quality. Normally, fruits grow and undergo three main maturity stages; maturation, ripening, and senescence with aerobic respiration. After the fruits reach maturation, a series of dramatic physiological and biochemical changes occur in the fruits, influencing color, flavor, and texture. ${ }^{1,2}$ Molecular oxygen is a biologically active molecule, and its concentration in fruits is of crucial importance for the maturation and ripening processes and for the quality of the fruits. Oxygen availability affects the respiratory metabolism. By decreasing the oxygen concentration and increasing the carbon dioxide concentration, the rate of the normal aerobic respiration process in which oxygen is consumed and carbon dioxide is produced decreases. ${ }^{3,4}$ Then a prolonged fruit lifetime results from a decreased respiration rate. However, carbon dioxide at a too high concentration can cause damage to the fruit tissue. ${ }^{4}$ Thus, it is of great interest and significance to optimize the gas concentrations during the postharvest time of fruits, especially for fruit preservation or storage.

Prolongation of the postharvest lifetime and inhibition of the physiological deterioration for fruits are priorities in fruit

*Address all correspondence to: Sune Svanberg, E-mail: sune.svanberg @fysik .Ith.se storage. Many techniques have been developed to address these issues, including controlled atmosphere (CA) storage and modified atmosphere packaging (MAP). Both CA storage and MAP are based on changing the gas composition to be different from that of ambient air, especially to significantly lower the oxygen concentration, to achieve a state of low physiological activity of fruits or vegetables, which would result in a prolonged shelf-life. The modified atmosphere slows down the chemical breakdown and microbiological spoilage of fruits. The main difference between CA storage and MAP is that in CA the composition of the surrounding atmosphere in the storage room is actively regulated, while in MAP the fruits are packed in an initially modified atmosphere, which will further change in a desired way due to the respiration metabolism of the enclosed fruits. Usually in MAP, carbon dioxide $\left(\mathrm{CO}_{2}\right)$ or nitrogen $\left(\mathrm{N}_{2}\right)$ are common substituted gases replacing part of the very reactive molecular oxygen in order to ensure quality and extension of the storage period of the fresh products. However, when the oxygen concentration is below a critical level, unwanted fermentation will be initiated. ${ }^{5} \mathrm{CO}_{2}$ behaves like an antimicrobiological gas, which prevents the growth of aerobic bacteria. Such growth is even more prevented at low temperatures, since water and lipids dissolve $\mathrm{CO}_{2}$ much better at low temperatures. $\mathrm{N}_{2}$, as a more inert gas, does not dissolve well in lipids or water, thus ensuring that a package still looks filled and is not collapsing. In MAP, a combination of high nitrogen and carbon dioxide concentrations is frequently used. Packaging with an oxygen concentration low enough to slow down the respiration and the maturation, but higher than the critical concentration for initiating fermentation, is an ideal one. However, since fruits continue to respire after harvest, MAP could in some cases cause anaerobic conditions $\left(\mathrm{O}_{2}<2 \%, \mathrm{O}_{2}>20 \%\right){ }^{6}$ resulting in increased anaerobic respiration. Due to this fact, high oxygen application in MAP was

$1083-3668 / 2016 / \$ 25.00$ @ 2016 SPIE 
introduced for the packaging of fresh-cut fruits and vegetables in 1996. ${ }^{7,8}$ Since that time, high oxygen MAP has been applied for such produce, including peppers, ${ }^{8}$ melons, ${ }^{9}$ pears, ${ }^{10}$ and lettuce. ${ }^{11}$

With MAP technology, fruits are frequently packed in plastic films. Films with different suitable permeability to different gases are used to optimize the concentrations of the gases in the packages. For optimal package design, there is a need for methods to measure and model the internal gas content and the gas exchange in fruits and packaging systems, especially noninvasively, to ensure quality and suitability for consumption. Techniques for assessing the gas content and gas exchange in the surrounding air have been developed. One method is to put the fruits into a closed container which is filled with a known gas mixture. After being closed for a while at a stable temperature, the gas exchange can be calculated from the changes of $\mathrm{O}_{2}$ and $\mathrm{CO}_{2}$ concentrations. In order not to initiate anaerobic respiration, fruits with high respiration rates should not be measured by this method. ${ }^{12}$ Another more common technique is to put the fruits into a flush-through system in which the gas exchange can be estimated from the concentration differences between the inlet and outlet gas flows. However, high gas flows from the fruits are needed in order to achieve a sufficient precision. ${ }^{13,14}$ Consequently, this technique is not suitable for fruits with low respiration rates. ${ }^{12}$ If the concentration differences are small, sensitive detection techniques are needed for more accurate assessments. Laser spectroscopy of gases emitted from fruits is a powerful and accurate method. ${ }^{15}$ For the internal gas contents and gas exchange measurements, techniques based on intrusive electrode probes or extraction of gas from the fruit interior by syringes are commonly used. ${ }^{16,17}$ The techniques described can be used to measure gas contents and gas dynamics but are frequently complicated or intrusive, thus influencing the fruit tissue which might affect the physiological processes.

Recently, there has been a strong development in the modeling of gas exchange in fruits, ${ }^{18-20}$ where experimental structural data have also been helpful, using, e.g., synchrotron radiation $\mathrm{x}$ ray microimaging. ${ }^{21}$ This has led to an increased understanding of the complex processes of gas exchange in fruits, including the presence of internal gradients.

Although there have been several experimental techniques for gas studies related to fruits, there is clearly room for additional and improved methods. A nonintrusive, compact, in-situ, and easily implemented spectroscopic technique-gas in scattering media absorption spectroscopy (GASMAS) - was introduced in 2001,22 and since then this technique has been developed into different applications, ${ }^{23,24}$ such as for wood, ${ }^{25-27}$ fruits, ${ }^{5,28,29}$ food packaging, ${ }^{30,31}$ and medical diagnostics. ${ }^{32,33}$ This technique employs a narrowband tunable diode laser to measure gases in pores and cavities in condensed-matter matrices. Here, we focus on oxygen content and gas exchange measurements in fruits, although other gases such as carbon dioxide and water vapor can be considered. GASMAS relies on the fact that free gases typically have 10,000 times narrower absorption features than the surrounding tissue. The GASMAS imprint is quite small-which is compensated for by using wavelength modulation spectroscopy (WMS) for high sensitivity detection. Since the probing light propagates through the fruit tissue and its interface to the outside world, the skin, the optical properties regarding absorption and scattering of fruit tissue are important and have recently been studied. ${ }^{34-37}$

The present investigations focus on the role of the skin in regulating gas transport in fruits, and oxygen diffusion measurements on peeled and unpeeled fruits were performed. The influence of the skin was studied for apples and guavas. Some results on apples were presented in a conference proceeding. ${ }^{38}$ Further, the gas exchange rate at different ripening stages was also studied in intact guavas by observing the transition from internal high-oxygen levels to the ambient gas concentration. The present work studying the gas dynamics greatly extends our earlier GASMAS studies of static gas behavior, as reported in Ref. 29, and also connects to the techniques of direct application of an artificial "skin" on the fruits, a surface coating. ${ }^{39}$

\section{Experimental Setup}

In most of our measurements, a distributed feedback diode (DFB) laser (LD-0760-0100, Toptica) with a nominal output power of $1.5 \mathrm{~mW}$ was used as the light source to generate the probing light. In some of the recordings, a higher power unit $(5 \mathrm{~mW})$ could be utilized. A laser temperature controller (TED200C, Thorlabs) and a laser current controller (LCD201C, Thorlabs) were used to control the drive temperature and current of the DFB diode laser to achieve wavelength tuning (Fig. 1). The diode laser was scanned across an $\mathrm{O}_{2}$ absorption line at $760.445 \mathrm{~nm}$ (vacuum wavelength) by sweeping the operating current of the laser using a $5 \mathrm{~Hz}$ sawtooth ramp. In order to perform sensitive WMS by using lock-in amplification techniques, the diode laser injection current was also modulated by a sinusoidal wave at $10,295 \mathrm{~Hz}$. An optical fiber with a $600-\mu \mathrm{m}$ core diameter (727-733-2447, Ocean Optics) was used to guide the light to the sample under study. Considering absorption and scattering when light passes through the sample, only a very small fraction of the light leaving the fiber could be detected in our study. A wavelengthmatched and large-area PMT with a 10-mm diameter (H10722-01, Hamamatsu) was chosen to detect the diffusely emerging light, which contains a weak absorption signal due to the oxygen inside the sample. Ambient light was suppressed by the combination of a colored glass long-pass filter (RG715, Edmund), and the photomultiplier tube quantum efficiency fall-off in the near-IR region, and also by using several black shields to suppress the ambient light. The signal from the photomultiplier tube was sent to a transimpedance amplifier (DLPCA-200, Femto) to yield a voltage signal, which was fed to a data acquisition (DAQ) card (PCI-6120, NI) which was also used for generation of the modulation signal for the DFB diode laser. The data from the DAQ card were stored in a computer for later processing. The whole system was controlled by a LabView program. The lock-in detection and signal processing procedure of the WMS signal were performed digitally with a MATLAB program. The WMS signal resembles the second derivative of the true absorption line shape when detected at twice the modulation frequency. Such recordings are referred to as $2 \mathrm{f}$ WMS signals. The wavelength modulation amplitude is typically chosen to be around half the absorption signal full half-width to maximize the signal while still obtaining a curve which is derivative-like. A more detailed description of the signal processing can be found in Refs. 40 and 41 .

\section{Samples}

Five Fuji apples, [as shown in Fig. 2(a)] for the fruit skin study, were acquired from the same batch at a local fruit shop in Southern China. Five guavas [as shown in Fig. 2(b)], also belonging to a specific batch, were bought in another fruit shop. Apples as well as guavas are climacteric fruits, 


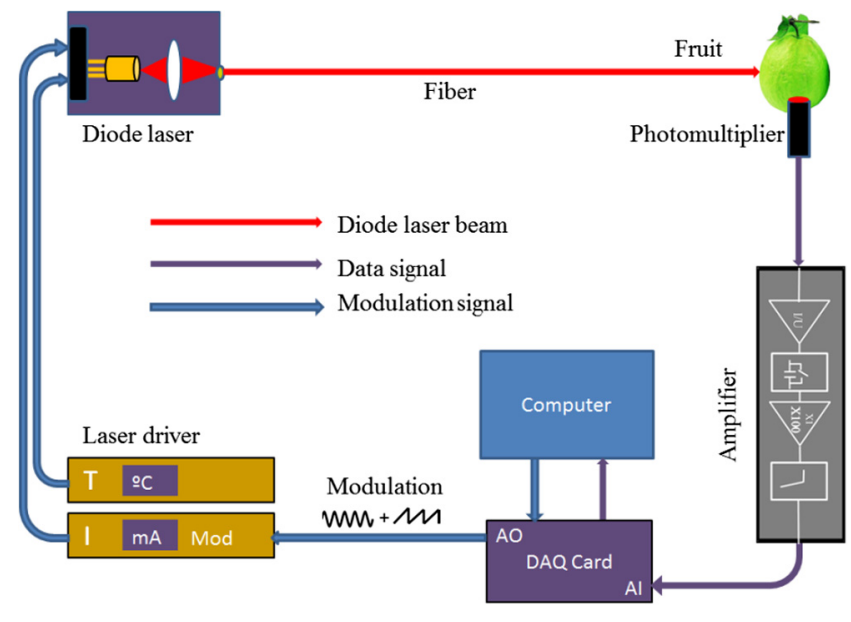

Fig. 1 Schematic layout of the GASMAS setup used for the $2 f$ WMS signal recording for oxygen.

characterized, e.g., by the maturation and ripening processes also continuing after harvest. Each apple or guava was studied regarding gas exchange, first unpeeled and the following day in a peeled state. Thus, each apple or guava experienced two different experiments during two consecutive days. Before the measurements, the samples were placed overnight in a sealed plastic bag filled with pure oxygen gas. Each peeled or nonpeeled apple or guava was placed in a separate transparent and sealed plastic bag for two consecutive days, and the bag was marked with different prefixes and numbers (e.g., Apple_1, Apple_2, etc. or Guava_1, Guava_2) matched to the samples for identification. For the fruit ripening case, three guavas were chosen from the same batch at the same local shop. Each of the three guavas was marked with a label for identification. Before the measurement day, the samples were left overnight in their identical transparent, sealed plastic bags which were filled with pure oxygen. The measurements took place over a 9 day span to see the change of gas dynamics in the ripening process, with measurements on days 1,5 , and 9 . The measurements were immediately started as soon as the sample fruit was taken out of the sealed oxygen bag. After some time of measurement, the oxygen signal came to a final equilibrium state, which meant that the gas exchange process was finished. The measurements were performed along the "equator" of the fruit in a "backscatteringlike" geometry with an injection-detection angular separation of 90 deg, as can be seen in Fig. 2(c). It was ascertained that the distance from the injection fiber to the center of the detector was kept constant at $5.0 \mathrm{~cm}$.

\section{Measurements and Results}

Because of the strong scattering properties of the fruits, we do not have a well-defined optical path length that light travels through the samples as required by the Beer-Lambert law to determine the exact oxygen concentration. Instead, we measure the equivalent mean path length $\left(L_{\text {eq }}\right)$ related to ambient air with a well-known $21 \%$ oxygen component, resulting in the same fractional absorption imprint on the received light intensity. $L_{\text {eq }}$ is proportional to the normalized WMS signal (i.e., the $2 \mathrm{f}$ WMS signal amplitude divided by the light intensity received by the detector; see further below). Thus, it is convenient and reliable to use $L_{\text {eq }}$ to estimate the oxygen content and the changes of oxygen contents in our study. Before the sample measurements, a single large absorption signal from the ambient air, with a known distance (in our study, a distance of $1200 \mathrm{~mm}$ is used), was utilized as the reference signal in order to extract $L_{\text {eq }}$ from the later sample signal by fitting.

The absorption signal due to gas in the scattering medium is very small; and WMS is used to improve the detection sensitivity. The size of the $2 \mathrm{f}$ WMS signal depends both on the detected light level and the gas absorption. Thus, the $2 \mathrm{f}$ WMS signal should be normalized to the amount of light detected in order to compensate for variations in the detected light intensity. This can be made by direct measurement of the light level hitting the detector, or by using the 1f WMS signal (also generated in the digital lock-in processing), the amplitude of which is proportional to the light level. ${ }^{41}$ The normalized GASMAS signal is thus a measure of the absorption due to the gas of interest, and is proportional to the absorbance, which is the product of the gas concentration and the path length traveled by the light. An example of an acquired $2 \mathrm{f}$ signal of a fruit is given in Fig. 3, where the experimental $2 \mathrm{f}$ signal (illustrated in blue color) has been fitted to a high quality reference line shape (illustrated in red color).

After prolonged exposure to pure oxygen, the fruit was taken out and GASMAS measurements were immediately initiated. The time evolution of the gas exchange was then followed, as illustrated in Fig. 4 for a peeled apple (a) and an intact and subsequently peeled guava (b). By fitting an exponential function to the experimental data with a MATLAB program, the initial $L_{\text {eq }}$, the final equilibrium $L_{\mathrm{eq}}$, and the time constant $\tau$ for the gas exchange dynamics were evaluated. Here, $\tau$ is the time it takes to change the signal initial value by a factor of $1 / \mathrm{e}$ in relation to the final equilibrium value. The corresponding values are given in the figure. A time constant of $24 \mathrm{~min}$ was found for the peeled apple, while the corresponding values for the intact and peeled guava are 6 and $15 \mathrm{~min}$, respectively. We note that the final $L_{\text {eq }}$ value in all cases is about 4 times the geometrical distance between the gas injection and detection points, illustrating (a)

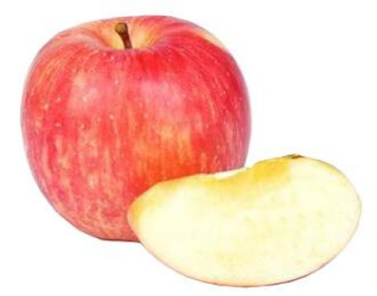

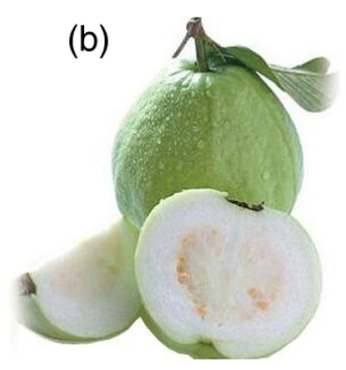

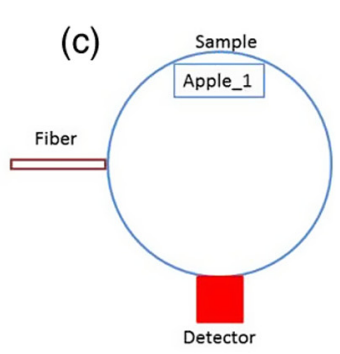

Fig. 2 (a) Fuji apples and (b) guavas are studied in the experiments. A schematic drawing of the measurement locations on the fruit skin along the fruit "equator" for the GASMAS probing is shown in (c). 


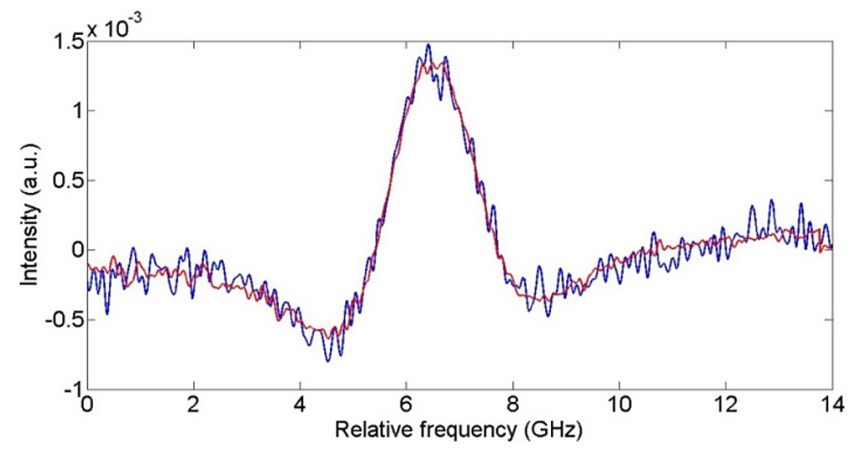

Fig. 3 Measured $2 f$ signals from a fruit (blue) and the fitted curve (red). The reference $2 f$ WMS oxygen signal used for fitting was attained for a distance of $1200 \mathrm{~mm}$ in ambient air. The signal shape resembles that of the second derivative of the gas absorption signal.

the strong multiple light scattering/diffuse light propagation typical for GASMAS. In this way, a representative sampling of the fruit interior is obtained, considering also that the gas distribution in fruits could also be inhomogeneous with gradients. $^{19,20}$

The time constant $\tau$ for returning to ambient conditions in the two different study conditions (unpeeled and peeled states) was measured for the five samples each for the two kinds of fruits, and the average $\tau$ values with error bars are shown in Figs. 5(a) and 5(c) for apple and guava, respectively. The spread in the data is partly due to the presence of residual interference fringes in the raw data, a typical phenomenon in diode laser spectroscopy. The expected function of the skin can be seen for apple, as the unpeeled apples exchange gas slower than the peeled ones. This confirms the assumption that the major site of resistance to gas exchange in fruits is the $\operatorname{skin}^{42}$ and agrees with the results for a single apple as presented in Ref. 5. In contrast, and quite surprisingly, we find that the opposite is true for guava: removing the skin leads to a longer time constant. As shown in Figs. 5(b) and 5(d), the final equilibrium $L_{\text {eq }}$ values for intact and peeled fruits behave oppositely for the apples and guavas, with the peeled apples having a larger value than the intact ones, while the opposite is observed for guavas. However, the trends are not very prominent in this case. It could be argued that the removal of the skin could influence the scattering conditions, and thus the effective path length for light traveled through the fruits. From Ref. 36, it is known that the scattering of apple skin is three times higher than the scattering from apple tissue. The
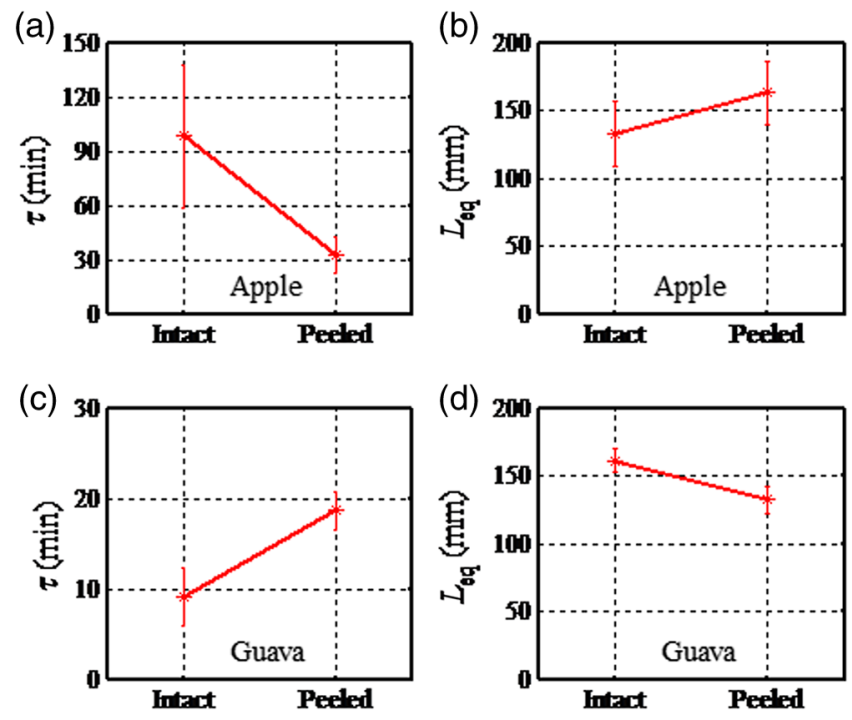

Fig. 5 (a)-(c) Average time constant $\tau$ of gas exchange for the groups five apples and five guavas in intact and peeled states, respectively. (b)-(d) Corresponding average final equilibrium oxygen values $L_{\text {eq }}$ for the apples and guavas experiencing gas exchange, respectively.

removal of the strong scatterer might be expected to reduce the effective path length through the apple, leading to a lowered final $L_{\text {eq }}$ for the peeled apple. However, we observe just the opposite, a larger $L_{\mathrm{eq}}$ for that case. This would suggest that the oxygen concentration has rather increased in the peeled apple. For guava, where the skin scattering is unknown, we observe the opposite trend.

Guava belongs to the tropical fruit family, and apple belongs to the group of temperate fruits. It is known that the respiration rate is higher for tropical fruits. In addition, guavas have a looser texture than apples and the content of the gas should be higher than in apples. We note that the equilibrium value for oxygen is higher for intact guava than for intact apple [see Figs. 5(d) and 5(b)]. However, it should be remembered that the scattering is also different for the two types of fruits.

One explanation why guavas behave differently from apples may be that guavas have a high respiration rate as a tropical fruit, and the skin helps the intact fruit to exchange gas quicker and easier than for the cold climate apple. This might also support the guava to achieve a relatively high oxygen concentration inside. The apple skin is relatively resistant to gas exchange and
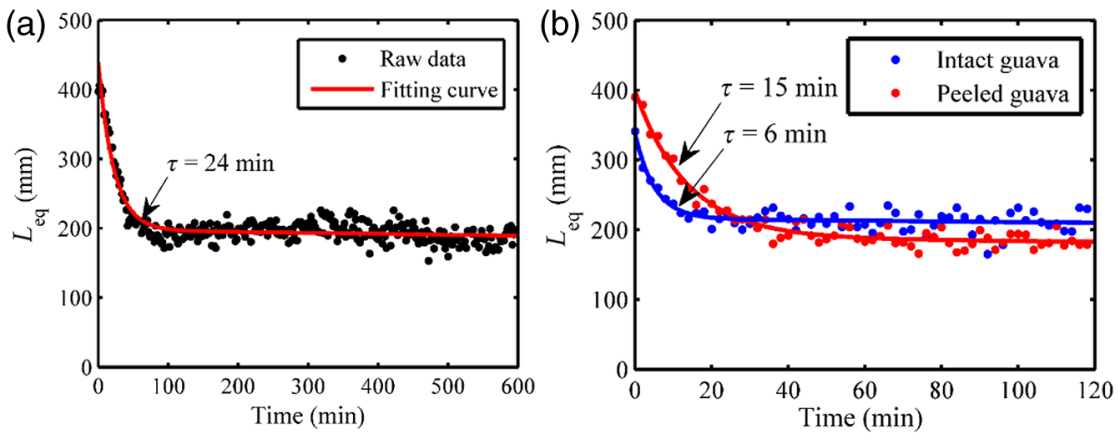

Fig. 4 (a) Example of gas exchange dynamics when a peeled apple returns to equilibrium after prolonged exposure to oxygen. (b) Corresponding curves for a guava, first in intact conditions ( $\tau=5 \mathrm{~min}$ ), and after peeling $(\tau=15 \mathrm{~min})$. Each data point corresponds to the recording of a curve of the type shown in Fig. 3 , and the $L_{e q}$ value is proportional to the fitted amplitude of curve. 

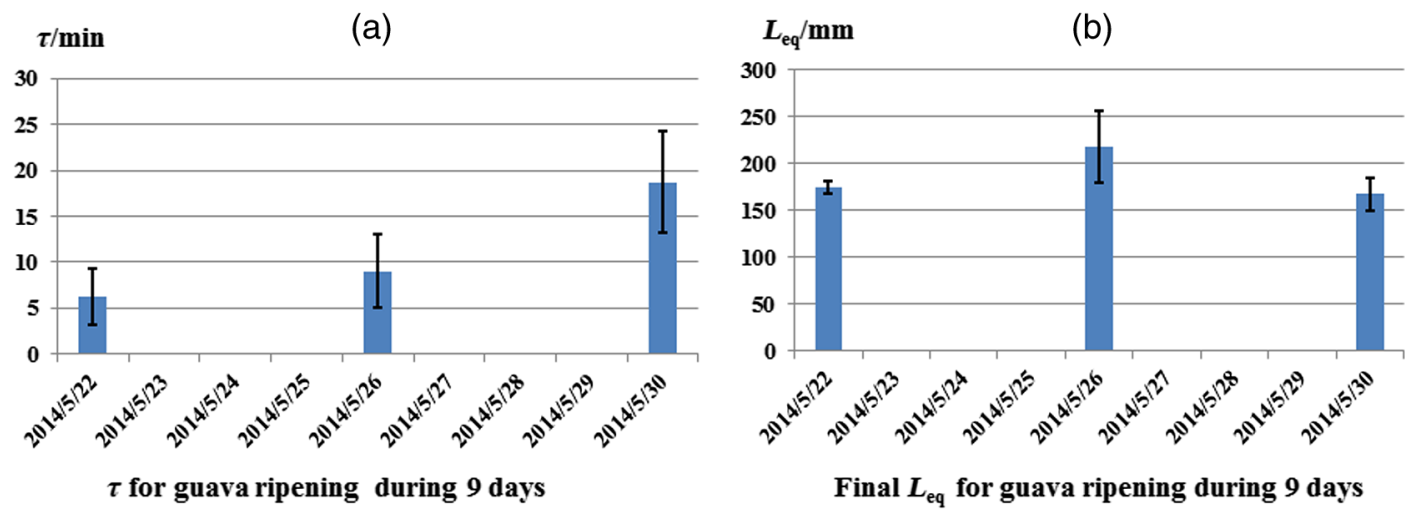

Fig. 6 (a) Average time constant $\tau$ after exposure to oxygen for three intact guavas during 9 days. (b) Average final equilibrium $L_{\text {eq }}$ for three guavas after experiencing gas exchange during 9 days.

thus keeps it in a lower respiration rate characteristic for temperate fruits. A different possibility could be that the normal fruit tissue of guava reacts in a protective way similar to that after mechanical bruises and then produces a barrier of cells which become lignified and which may block gas transport even more effectively than fruit skin.

An interesting observation in this study was that neither the peeled apples nor the peeled guavas turned brownish during the high oxygen concentration exposure in the closed bags. Further, it took almost 2 days or an even longer time for the peeled fruits to turn brown in subsequent ambient air conditions. Also, in Fig. 5, it is clearly seen that the peeled guavas kept in a high oxygen environment decreased the gas exchange rate, which should result in a prolonged shelf-life. The implication might be that fresh-cut guava should be stored in high oxygen MAP.

Our results from the study of gas contents and gas exchange during ripening are shown in Fig. 6. From Fig. 6(a), it can be seen that the time constant $\tau$ just changed slightly during the first 5 days, while at day 9 the time constant $\tau$ is much larger than before. An explanation could be that at the beginning the guava as a tropical fruit respires at a high rate and, therefore, the time constant $\tau$ is fast. When time passes by, the guava slows down the respiration resulting in a slow down of the time constant $\tau$ as observed at day 9. During the first 4 days, the guavas were visibly still fresh with relatively high respiration, but at day 9 , the guavas were decaying with a relative low respiration. Tropical fruits are usually connected with high respiration rates, which means that they have fast metabolism; however, the more intense the metabolism is, the quicker the ripening of the fruits will be, and the shorter the shelf-time. As can be seen in Fig. 6(b), the $L_{\text {eq }}$ inside the guavas also changed during the ripening period with an increase in the beginning and then a decrease, which means that the detected signal due to the absorption of the gas of interest changed. The normalized GASMAS signal is always the product of concentration and path length; thus the changes in $L_{\text {eq }}$ shown in Fig. 6(b) can come from the changes in concentration or in path length through the gas or both. One interpretation for the variation of $L_{\text {eq }}$ might be that the respiratory rate changes during the fruit ripening. Since guava is a climacteric fruit, once it begins ripening, its respiratory rate increases until it reaches a peak, after which the respiratory rate decreases, finally to cease. The peak value, sometimes called the climacteric point, relates to the fruit ripening associated with a maximum in respiration rate and a burst of ethylene. ${ }^{43,44}$ Assuming constant scattering, we note from Fig. 6, that before the guava reaches ripening, it respires relatively high with high oxygen production, to reach the climacteric point after which the respiration rate slows down. However, it should be borne in mind that during the ripening process, the optical properties most likely change for guava, as was actually noted in studies of the likewise climacteric fruit pear. ${ }^{45}$ This makes the conclusions regarding oxygen contents uncertain. The product of effective path length and concentrations as measured by GASMAS is, however, unaffected by this, and can still be used to characterize the fruit during the storage period. It is important to note that the time constants measured with GASMAS, as well as gas pressure ${ }^{28}$ and temperature, ${ }^{46}$ are unaffected by changing path lengths.

In view of the unexpected skin-action results obtained for guava, with a longer gas-exchange constant in the peeled state, a new series of measurements on a different batch of guavas were made on a later occasion. The results were verified-indeed peeling of the guava leads to slower gas exchange. Actually, the curves shown in Fig. 4(b) refer to these latter control measurements. As a further check, we performed oxygen GASMAS measurements following prolonged immersion of the guava fruit in pure nitrogen, and observing the subsequent relaxation back to ambient conditions. Now the signal-to-noise situation is challenging since we now work with an oxygen concentration which at most was ambient $(21 \%)$ instead of $100 \%$ as in the main part of the experiment. Also, when the physiologically less active nitrogen gas was used, a longer time constant ( $25 \mathrm{~min}$ to compared $13 \mathrm{~min}$ ) was obtained for the peeled fruit. It should be noticed that the measurements of time constants are not affected by scattering, which is otherwise the case when measuring $L_{\mathrm{eq}}$ values, which always display the product of concentration and effective, scatter-influenced path length.

\section{Conclusions}

In the present paper, nonintrusive measurements of gas exchange through intact and peeled apple and guava fruits were demonstrated using the GASMAS technique, by observing the response of the oxygen equivalent mean path length to a transient change in the ambient gas environment. The development of gas transport during the maturing process was also studied. In contrast to other techniques, the gas is measured inside the fruit tissue in a completely nonintrusive way. We note that while equivalent path length values also depend on scattering and not only on gas concentration, this is not the case for the time constant determinations, which is also true for pressure ${ }^{28}$ and 
temperature ${ }^{46}$ measurements. It was observed that the fruit skin has a strong influence on gas exchange dynamics. While the temperate apple behaves in an intuitive way with its fruit skin slowing gas exchange, the tropical fruit guava unexpectedly behaves in the opposite way. It would be interesting to verify this behavior using conventional techniques and further elucidate its origin. Our study suggests that the GASMAS technique might be developed into a valuable, noninvasive, and real-time tool for gas and gas exchange studies in fruits and in plant physiology in general, as well as for a broad range of applications in food storage and packaging.

\section{Disclosures}

The authors state that they have no conflicts of interest related to the work presented.

\section{Acknowledgments}

The authors are thankful to Yuan Fu for his assistance in setting up the GASMAS system. We are also thankful for Professor Sailing He for his kind support. This work was supported by a Guangdong Province Innovation Research Team Program (No. 201001D0104799318).

\section{References}

1. A. A. Kader, "Fruit maturity, ripening, and quality relationships," Acta Hortic. 485, 203-208 (1999).

2. J. M. Palma, F. J. Corpas, and L. A. del Río, "Proteomics as an approach to the understanding of the molecular physiology of fruit development and ripening," J. Proteomics 74, 1230-1243 (2011).

3. R. M. Beaudry, "Effect of $\mathrm{O}_{2}$ and $\mathrm{CO}_{2}$ partial pressure on selected phenomena affecting fruit and vegetable quality," Postharvest Biol. Technol. 15, 293-303 (1999).

4. M. L. A. T. M. Hertog et al., "A dynamic and generic model of gas exchange of respiring produce: the effects of oxygen, carbon dioxide and temperature," Postharvest Biol. Technol. 14, 335-349 (1998).

5. L. Persson et al., "Diode laser absorption spectroscopy for studies of gas exchange in fruits," Opt. Lasers Eng. 44, 687-698 (2006).

6. L. Jacxsens, F. Devlieghere, and C. van der Steen, "Effect of high oxygen modified atmosphere packaging on microbial growth and sensorial qualities of fresh-cut produce," Int. J. Food Microbiol. 71, 197-210 (2001).

7. B. P. F. Day, "High oxygen modified atmosphere packaging for fresh prepared produce," Postharvest News Inf. 7, 31-34 (1996).

8. A. Conesa, F. Artes-Hemandez, and S. Geysen, "High oxygen combined with high carbon dioxide improves microbial and sensory quality of fresh-cut peppers," Postharvest Biol. Technol. 43, 230-237 (2007).

9. G. Oms-Oliu, M. R. M. Raybaudi-Massilia, and R. Soliva-Fortuny, "Effect of superatmospheric and low oxygen modified atmospheres on shelf-life extension of fresh-cut melon," Food Control 19, 191199 (2008).

10. G. Oms-Oliu, R. Soliva-Fortuny, and O. Martin-Belloso, "Physiological and microbiological changes in fresh-cut pears stored in high oxygen active packages compared with low oxygen active and passive modified atmosphere packaging," Postharvest Biol. Technol. 48, 295-301 (2008).

11. V. H. Escalona, B. E. Verlinden, and S. Geysen, "Changes in respiration of fresh-cut butterhead lettuce under controlled atmospheres using low and superatmospheric oxygen conditions with different carbon dioxide levels," Postharvest Biol. Technol. 39, 48-55 (2006).

12. X. Y. Wang et al., "Advances of respiration rate in modified atmosphere packaging for fruits and vegetables," Trans. Chin. Soc. Agric. Mach. 39, 94-100 (2008).

13. G. Andrich et al., "Automatic control of aerobic and anaerobic respiration rates of fruits stored in refrigerated and controlled atmospheres," in Automatic Control of Food and Biological Processes, J. J. Bimbenet, Ed.,pp. 281-287, Elsevier, Amsterdam (1994).
14. P. Varoquaux, G. Albagnac, and B. Gouble, "Physiology of minimally processed fresh vegetables," in Australasian Postharvest Horticulture Conf., pp. 437-443 (1995).

15. D. D. Arslanov et al., "Rapid and sensitive trace gas detection with continuous wave optical parametric oscillator-based wavelength modulation spectroscopy," Appl. Phys. B 103, 223-228 (2011).

16. B. K. Dadzie et al., "Changes in respiration and ethylene production of apples in response to internal and external oxygen partial pressures," Postharvest Biol. Technol. 9, 297-309 (1996).

17. J. Oomens et al., "CO-laser-based photoacoustic trace-gas detection: applications in postharvest physiology," Appl. Phys. B Lasers Opt. 67, 459-466 (1998).

18. Q. T. Ho et al., "Genotype effects on internal gas gradients in apple fruit,” J. Exp. Bot. 61, 2745-2755 (2010).

19. Q. T. Ho et al., "A three-dimensional multiscale model for gas exchange in fruit," Plant Physiol. 155, 1158-1168 (2011).

20. Q. T. Ho et al., "A multiphase pore scale network model of gas exchange in apple fruit," Food Bioprocess Technol. 7, 482-495 (2014).

21. P. Verboven et al., "Three-dimensional gas exchange pathways in pome fruit characterized by synchrotron x-ray computed tomography," Plant Physiol. 147, 518-527 (2008).

22. M. Sjöholm et al., "Analysis of gas dispersed in scattering media," Opt. Lett. 26, 16-18 (2001).

23. S. Svanberg, "Analysis of trapped gas-gas in scattering media absorption spectroscopy," Laser Phys. 20, 68-77 (2010).

24. S. Svanberg, "Gas in scattering media absorption spectroscopy-from basic studies to biomedical applications," Laser Photonics Rev. 7, 779796 (2013).

25. J. Alnis et al., "Laser spectroscopy of free molecular oxygen dispersed in wood materials," Appl. Phys. B 77, 691-695 (2003).

26. M. Andersson et al., "Spectroscopic studies of wood-drying processes," Opt. Express 14, 3641-3653 (2006).

27. I. Bargigia et al., "Diffuse optical techniques applied to wood characterization," J. Near Infrared Spectrosc. 21, 259 (2013).

28. U. Tylewicz et al., "Gas in scattering media absorption spectroscopy (GASMAS) detected persistent vacuum in apple tissue after vacuum impregnation," Food Biophys. 7, 28-34 (2011)

29. H. Zhang et al., "Studies of tropical fruit ripening using three different spectroscopic techniques," J. Biomed. Opt. 19, 067001 (2014).

30. M. Lewander et al., "Food monitoring based on diode laser gas spectroscopy," Appl. Phys. B 93, 619-625 (2008).

31. M. Lewander et al., "Non-intrusive measurements of headspace gas composition in liquid food packages made of translucent materials," Packag. Technol. Sci. 24, 271-280 (2011).

32. L. Persson et al., "Gas monitoring in human sinuses using tunable diode laser spectroscopy," J. Biomed. Opt. 12, 054001 (2007).

33. M. Lewander et al., "Noninvasive diagnostics of the maxillary and frontal sinuses based on diode laser gas spectroscopy," Rhinology 50, 28-32 (2012).

34. R. Cubeddu et al., "Measuring fresh fruit and vegetable quality: advanced optical methods," Chapter 8 in Fruit and Vegetable Processing, W. Jongen, Ed., pp. 150-169, Woodhead Publishing, Cambridge (2002).

35. B. M. Nicolai et al., "Nondestructive measurements of fruit and vegetable quality by means of NIR spectroscopy: a review," Postharvest Biol. Technol. 46(2), 99-118 (2007).

36. W. Saeys et al., "Optical properties of apple skin and flesh in the wavelength range from 350 to $2200 \mathrm{~nm}$," Appl. Opt. 47, 908-919 (2008).

37. A. Torricelli et al., "Recent advances in time-resolved NIR spectroscopy for nondestructive assessment of fruit quality," Chem. Eng. Trans. 44, 43-48 (2015).

38. J. Huang et al., "Studies of oxygen and oxygen exchange in fruits using gas in scattering media absorption spectroscopy," in Proc. Progress in Electromagnetics Research Symp. (PIERS), Vol. 2014, pp. 1251-1255 (2014).

39. N. H. Banks, B. K. Dadzie, and D. J. Cleland, "Reducing gas exchange of fruits with surface coatings," Postharvest Biol. Technol. 3, 269-284 (1993).

40. T. Svensson et al., "VCSEL-based oxygen spectroscopy for structural analysis of pharmaceutical solids," Appl. Phys. B 90, 345-354 (2008).

41. L. Mei and S. Svanberg, "Wavelength modulation spectroscopy-digital detection of gas absorption harmonics based on Fourier analysis," Appl. Opt. 54, 2234-2243 (2015). 
42. A. C. Cameron, P. C. Talasila, and D. W. Joles, "Predicting film permeability needs for modified-atmosphere packaging of lightly processed fruits and vegetables," Hortic. Sci. 30, 25-34 (1995).

43. L. Alexander and D. Grierson, "Ethylene biosynthesis and action in tomato: a model for climacteric fruit ripening," J. Exp. Bot. 53, 2039-2055 (2002).

44. V. Paul, R. Pandey, and G. C. Srivastava, "The fading distinction between classical patterns of ripening in climacteric and non-climacteric fruit and the ubiquity of ethylenean overview," J. Food Sci. Technol. 49, 1-21 (2012).

45. A. Brázio, A. Cavaco, and R. Guerra, "A simplified two layer model for light diffuse reflectance in thin skin fruits," Prog. Agric. Eng. Sci. 6, 3572 (2010).

46. J. Larsson, S. Svanberg, and J. Bood, "Thermometry in porous media using GASMAS-a feasibility study," in Proc. LACSEA Conf. 2016, Heidelberg Germany, OSA, Washington, D. C. (2016).

Jing Huang received her bachelor's degree from Guangdong University of Technology, Guangzhou in 2012. She took her MSc exam at the Center for Optical and Electromagnetic Research at South China Normal University, Guangzhou, in 2015, and currently, she is a PhD student at Friedrich Schiller University, Jena, Germany. Her research interests include laser spectroscopy applied to food safety and biomedical topics.

Hao Zhang received his bachelor's degree in physics from East China Institute of Technology, Nanchang, in 2011. His major field was optical engineering. He defended his $\mathrm{PhD}$ at the Center for Optical and Electromagnetic Research at South China Normal University, Guangzhou, in 2016 and is now a post-doc at Henan Agricultural University, Zhengzhou. His research interests concern applications of laser spectroscopy to the biophotonics and agricultural fields.

Huiying Lin received her bachelor's degree from Guangdong University of Technology, Guangzhou, in 2014. She is now a master's student at the Center for Optical and Electromagnetic Research at South China Normal University, Guangzhou. Her research interests include laser spectroscopy applied to biomedical and food safety topics.

Tianqi Li received her bachelor's degree in applied physics at Hefei University of Technology in 2013. She had her MSc exam at the Center for Optical and Electromagnetic Research at South China Normal University, Guangzhou, in 2016. Her research interests include laser spectroscopy applied to food safety and biomedical topics.

Liang Mei obtained his $\mathrm{PhD}$ from Zhejiang University in 2013 and then from Lund University in 2014 with independent research topics. $\mathrm{He}$ is now affiliated with the School of Physics and Optoelectronic Engineering, Dalian University of Technology, where he has been active as an associate professor since 2015. His research interests concern applications of laser spectroscopy to environmental, biophotonics, and food safety fields.

Katarina Svanberg obtained her PhD from Lund University in 1989 and is affiliated with the Department of Oncology, Lund University Hospital, where she has been active as a chief consultant and professor of oncology since more than 25 years. Since 2011, she has also been a distinguished professor at South China Normal University, Guangzhou. Her research interests concern applications of laser spectroscopy to the biomedical and biophotonics fields.

Sune Svanberg obtained his PhD from University of Gothenburg in 1972, and since 1980, he has been a professor of physics at Lund University, Lund, Sweden. For 30 years, he was head of the Atomic Physics Division, and during 20 years, he was director of the Lund Laser Center. Since 2011, he has also been a distinguished professor at South China Normal University, Guangzhou. His research interests include laser spectroscopic applications to the environmental, food safety, and biomedical fields. 